Revista Digital Universitaria

Vol. 22, Núm. 6, noviembre-diciembre 2021

\title{
Micromundos biominerales en las algas rojas
}

\author{
Patricia M. Valdespino, Andrea Bautista, Teresa Pi-Puig, Fabio \\ Favoretto, Silvia Espinosa Matías, Hoi-Ying N. Holman y \\ Anidia Blanco-Jarvio
}

\section{Resumen}

Las algas rojas coralinas (rodofitas) habitan las costas, coloreando estas regiones con una hermosa tonalidad roja o rosada, y son tan duras y afiladas que, de ser pisadas, causarían dolor al pie descalzo. Entre sus diminutas ramas, muchos organismos se desarrollan y encuentran refugio. La fotosíntesis de las algas coralinas conduce a la formación de estructuras de carbonatos con una arquitectura fascinante. Pero tanto el alga como sus microorganismos asociados (microbiota) participan en la formación de estos biominerales, que se acumulan y cementan los materiales que forman las playas y las costas. Los carbonatos son susceptibles a la química ácido-base, por lo tanto, su estabilidad estructural y su disolución dependen del $\mathrm{pH}$ del medio que los rodea. Por tanto, estos biominerales y los organismos marinos que los forman (como algas, corales, moluscos o equinodermos) son vulnerables a la acidificación del océano.

La curiosidad por ver lo que nuestros ojos no pueden nos llevó a entender que las delicadas ramitas de las algas coralinas esconden una asombrosa fortaleza estructural donde la microestructura y la química son elementos clave, pues encontramos que minerales con una gran estabilidad estructural y térmica constituyen el cuerpo, o talo, de las algas. Hoy en día las algas rojas y verdes coralinas son modelos vivos de gran interés para la ciencia básica, para capturar carbono en estructuras estables. Así, esta área de investigación puede inspirar el desarrollo de estrategias para mitigar el cambio climático.

Palabras clave: biominerales, calcita magnesiana, ciclo del carbono, acidificación oceánica, biología de sistemas, sincrotrón.

\section{BIOMINERAL MICROWORLDS IN RED ALGAE}

\begin{abstract}
Coralline algae (rhodophyta) populate vast pinkish colour regions of the coast. If you step on them in your bare feet, they might hurt you, because they are hard and sharp. Many organisms find shelter and develop within their tiny branches. Photosynthesis of coralline algae conducts the formation of carbonates that exhibit a fascinating architecture. The alga and its associated microorganisms (microbiota) participate in the formation of these minerals, that accumulate and cement the materials that ultimately shape beaches and coastal lines. Carbonates are susceptible to acid-base chemistry; thus, their structural stability and their dissolution depend on the $\mathrm{pH}$ of the surrounding environment. Therefore, these biominerals and the marine organisms that build them (such as algae, corals, mollusks or equinoderms) are vulnerable to ocean acidification.

By trying to see beyond our eyesight, we were able to understand that algal branches hide an amazing structural strength, where its microstructure and chemistry play a major role. We found minerals with a vast structural and thermal stability in the algal body, named algal thallus. Currently, basic science explores coralline red and green algae as interesting models to understand carbon sequestration in stable structures. Therefore, this research might inspire the development of technologies to mitigate climate change.
\end{abstract}

Keywords: biominerals, magnesium calcite, carbon cycle, ocean acidification, systems biology, synchrotron.

Recepción: 08/12/2020. Aprobación: 13/04/2021.

Dol: http://doi.org/10.22201/cuaieed.16076079e.2021.22.6.4

Universidad Nacional Autónoma de México, Coordinación de Universidad Abierta, Innovación Educativa y Educación a Distancia (CUAIEED) Este es un artículo de acceso abierto bajo la licencia de Creative Commons 4.0 cc)(i)(2) 
"Micromundos biominerales en las algas rojas"

Patricia M. Valdespino, Andrea Bautista, Teresa Pi-Puig, Fabio Favoretto, Silvia Espinosa Matías, Hoi-Ying Holman y Anidia Blanco-Jarvio Vol. 22, Núm. 6, noviembre-diciembre 2021 Revista Digital Universitaria

Patricia M. Valdespino pancronica@gmail.com orcid.org/0000-0002-2998-4627 sites.google.com/view/pancronos/home

Investigadora postdoctoral en el Berkeley Lab en California (sNI- Nivel I). Estudió Biología en la Universidad Nacional Autónoma de México (UNAM), donde también realizó el posgrado en Ciencias del Mar y Limnología. Su investigación se enfoca en el estudio de los ciclos biogeoquímicos del planeta en interacción con la Biología de sistemas. Estudia ensambles microbianos acuáticos con estrategias tanto clásicas como de nueva generación, integrando la genómica microbiana con imagen química acoplada a luz de sincrotrón. Es una entusiasta de la ciencia y del arte como guías para trabajar por un mundo mejor.

Andrea Bautista García

bg.andrea93@gmail.com orcid.org/0000-0002-5426-287X

Es estudiante de Maestría en Ciencias Acuáticas en la uabcs en La Paz, México. Su proyecto de maestría se enfoca en la microestructura de las algas coralinas y en los microbios que las habitan. Es profesora y entusiasta divulgadora de la ciencia.

Teresa Pi-Puig

tpipuig@geologia.unam.mx orcid.org/0000-0002-0285-382X

Responsable del laboratorio de Difracción de Rayos X del Instituto de Geología y del Laboratorio Nacional de Geoquímica y Mineralogía (LANGEM) y profesor de Mineralogía en La Licenciatura y el Posgrado en Ciencias de la Tierra.

Su área de interés es la mineralogía, aplicando las herramientas de esta disciplina tanto a proyectos de ciencia básica como de ciencia aplicada en diferentes áreas temáticas como la cristalografía, identificación y caracterización de minerales, yacimientos minerales y geotermia, minerales y rocas industriales, y mineralogía de arcillas. Fruto de su participación en proyectos de infraestructura, ciencia básica y docencia ha contribuido en la publicación de numerosos artículos en revistas del scl.

Fabio Favoretto

favorettofabio@gmail.com orcid.org/0000-0002-6493-4254

Jefe científico del Center for Marine Biodiversity and Conservation, A.C. (сBMc). Professor at Universidad Autónoma de Baja California Sur (UABCS).

Su investigación en el área marina abarca las ciencias de los datos a través de una formación fuerte en ecología. Le interesa aplicar nuevas tecnologías para la conservación de los recursos naturales y la fauna silvestre.

Silvia Espinosa Matías

emfc2010@gmail.com https://orcid.org/0000-0002-7629-5816

Realizo sus estudios en la Facultad de Ciencias de la Universidad Nacional Autónoma de México (UnAm).

Responsable del Laboratorio de Microscopía Electrónica de Barrido de la misma Facultad. Ha desarrollado sus actividades académicas en el campo de la Microscopia Electrónica de Barrido y Botánica Estructural abarcando los rubros de Asesorías Técnicas, Docencia, Investigación y Difusión. 
Hoi-Ying N. Holman

hyholman@lbl.gov https://orcid.org/0000-0002-7534-2625

Directora del Programa de Biología Estructural por infrarrojo Acoplado a luz de Sincrotrón de Berkeley (www.bsisb.|lbl.gov) en el Lawrence Berkeley National Laboratory)

\section{Anidia Blanco-Jarvio}

ablanco@uabcs.mx orcid.org/0000-0002-1480-4988

Facebook: @BICA.UABCS

Investigadora Titular responsable del Laboratorio de Bioingeniería y Ciencias Ambientales (BICA) de la Universidad Autónoma de Baja California Sur (UABCS).

Ha buscado generar una visión multidisciplinaria e integral que dé respuestas a problemáticas ambientales, su investigación de manera específica en ecología microbiana se ha enfocado en el estudio de la diversidad y transformaciones metabólicas en diversos ecosistemas. 
"Micromundos biominerales en las algas rojas" Patricia M. Valdespino, Andrea Bautista, Teresa Pi-Puig, Fabio Favoretto, Silvia Espinosa Matías, Hoi-Ying Holman y Anidia Blanco-Jarvio Vol. 22, Núm. 6, noviembre-diciembre 2021 Revista Digital Universitaria

\section{Micromundos minerales en pequeñas ramitas}

1 uestra historia comienza en Baja California Sur, en las pozas intermareales o charcas en la Bahía de la Paz que se forman en las playas rocosas cuando baja la marea. Allí puedes encontrar a Neogoniolithon trichotomum, el alga coralina dominante. La reconocerás porque parece un bosque sumergido de hermosos árboles rosados del tamaño de tu mano, con ramas de aproximadamente de $1 \mathrm{~mm}$ de grosor (ver figura 1). Las algas coralinas son fascinantes y complejas, pero ahora nos enfocaremos en su composición mineral. Estas algas participan en la precipitación de una estructura mineral carbonatada, dura como los dientes. De hecho, de forma similar a los carbonatos identificados en las algas, los huesos en nuestro cuerpo están formados principalmente de otro biomineral Ilamado hidroxiapatita. Este mineral (acompañado de compuestos orgánicos como el colágeno) le da estructura a nuestro organismo y, a diferencia de los minerales que forman los esqueletos de las algas, no es un carbonato de calcio, es un fosfato de calcio $\left[\mathrm{Ca}_{5}\left(\mathrm{PO}_{4}\right)_{3}(\mathrm{OH})\right]$.

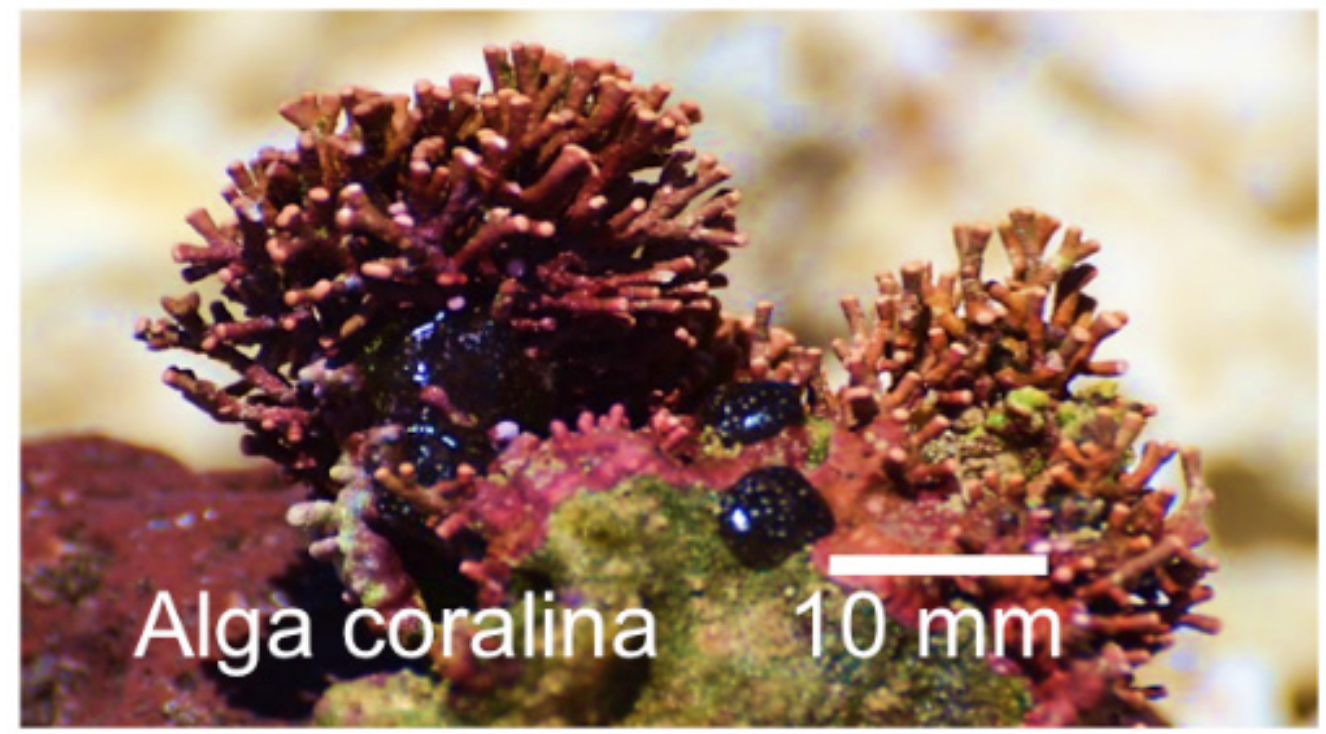

Para investigar los micromundos biominerales y cómo se organizan sus habitantes necesitamos un microscopio potente y una técnica de detección fina de los minerales. Así que, pedimos apoyo de expertos y usamos microscopía electrónica de barrido (conocida como microscopía SEM) y difracción de rayos- $X$ (DRX). Si te llama la atención ver lo que nuestros ojos no pueden, esto te puede interesar.

Las imágenes de microscopía SEm revelaron una microestructura de gran belleza. En la figura 2 se puede apreciar un corte lateral de la ramita. La zona superficial, con cavidades geométricas, y de paredes tan delgadas como una milésima parte de un milímetro (o micra), es la zona de crecimiento del alga, llamada peritalo. La zona más interna de las ramas muestra una estructura 
"Micromundos biominerales en las algas rojas" Patricia M. Valdespino, Andrea Bautista, Teresa Pi-Puig, Fabio Favoretto, Silvia Espinosa Matías, Hoi-Ying Holman y Anidia Blanco-Jarvio Vol. 22, Núm. 6, noviembre-diciembre 2021 Revista Digital Universitaria

Figura 2. Microestructura de las ramitas del alga coralina en vista lateral y vista superficial. Se distinguen la capa de crecimiento o peritalo y la capa superficial, que se curva y que se cubre de materiales y organismos como diatomeas (Laboratorio de Microscopía Electrónica de Barrido, Facultad de Ciencias, UnAm). mineral en la que las paredes carbonatadas son bastante más gruesas (casi 10 micras). También puedes observar que las cavidades carbonatadas contienen numerosos gránulos redondeados. Es difícil estudiarlos por su diminuto tamaño y se cree que son gránulos de almidón o habitantes microbianos. Sus detalles son difíciles de analizar dada su posición y su pequeño tamaño (alrededor de 2 micras). Sin embargo, la microscopía sEm nos reveló esta compleja microarquitectura (Bautista, 2020).

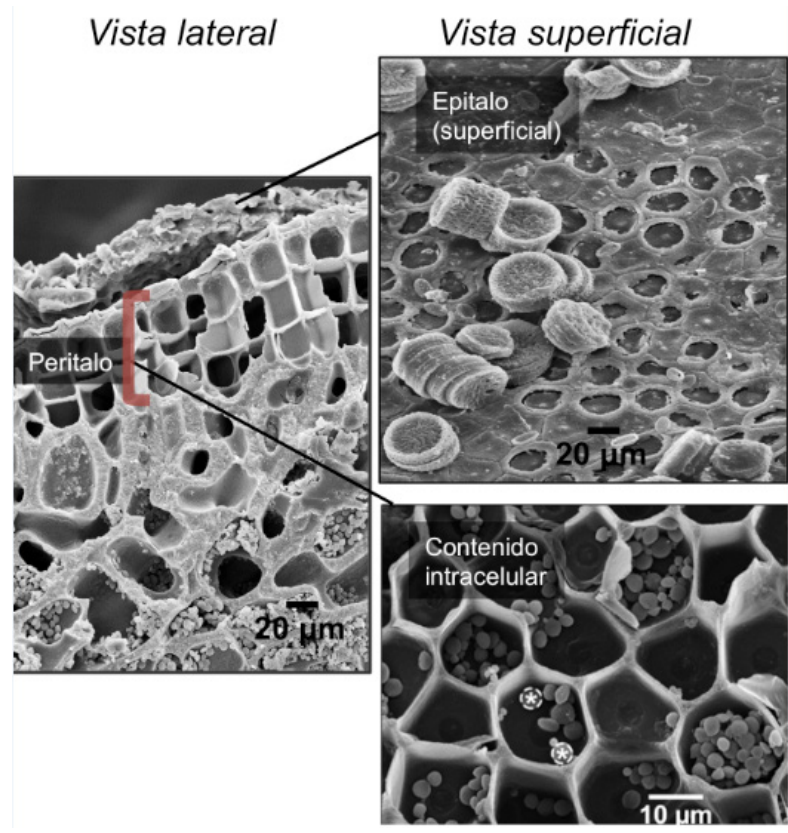

\section{¿Qué es la acidificación del océano y cómo amenaza a los organismos formadores de carbonatos?}

La formación y la disolución de los carbonatos está afectada por el pH del medio que los rodea. El agua de mar tiene en promedio un $\mathrm{pH}$ de 8.1, es decir, es ligeramente alcalino. Desde hace más de una década, se ha reportado en cientos de trabajos de investigación una tendencia hacia la acidificación del agua marina: es decir, hay disminución del $\mathrm{pH}$. Lo anterior ocurre porque con el aumento de la cantidad de dióxido de carbono $\left(\mathrm{CO}_{2}\right)$ en la atmósfera, dicho gas se difunde en mayor proporción hacia el agua, lo que incrementa la concentración de los iones $\mathrm{H}+$ y provoca, por tanto, una disminución del pH y el aumento de acidez del agua marina. Esto es relevante pues afecta los equilibrios químicos en los ambientes acuáticos.

Pero ¿cómo son afectados los carbonatos? Pues, de forma muy directa ya que se disuelven si el pH que los rodea es ácido. Este proceso ocurre de forma natural, por ejemplo, en las rocas carbonatadas de la península de Yucatán. Éstas se disuelven en un ambiente acuático alimentado por el agua de la lluvia y forman estructuras kársticas (generadas por meteorización química), como los famosos cenotes. Otro ejemplo es el hallazgo de la disolución de los carbonatos de las pequeñas conchas de los pterópodos o mariposas de mar. La disolución de los carbonatos puede ser un problema para muchos organismos marinos que fabrican esqueletos, conchas y dientes de carbonatos. Algunos ejemplos son las algas rojas, algas verdes, corales, moluscos (como caracoles y ostras), equinodermos (estrellas de mar y erizos), etcétera. 


\section{Investigación en ambientes costeros de la Bahía de la Paz, Baja California Sur}

El grupo de investigación de Bioingeniería y Ciencias Ambientales (BICA) de la Universidad Autónoma de Baja California Sur (UABCS) realiza un monitoreo periódico de las pozas intermareales de la Bahía de La Paz, desde hace algunos años (ver figura 3). Mientras que distintos miembros del equipo estudian sistemas biológicos como los bivalvos o los crustáceos, el proyecto de Maestría de Andrea se enfocó en estudiar detalladamente las algas coralinas.

Figura 3. Área de estudio en la Bahía de La Paz, Baja California Sur.

Figura 4. Vista desde el aire de las pozas intermareales estudiadas (cortesía de Hiram Rosales).

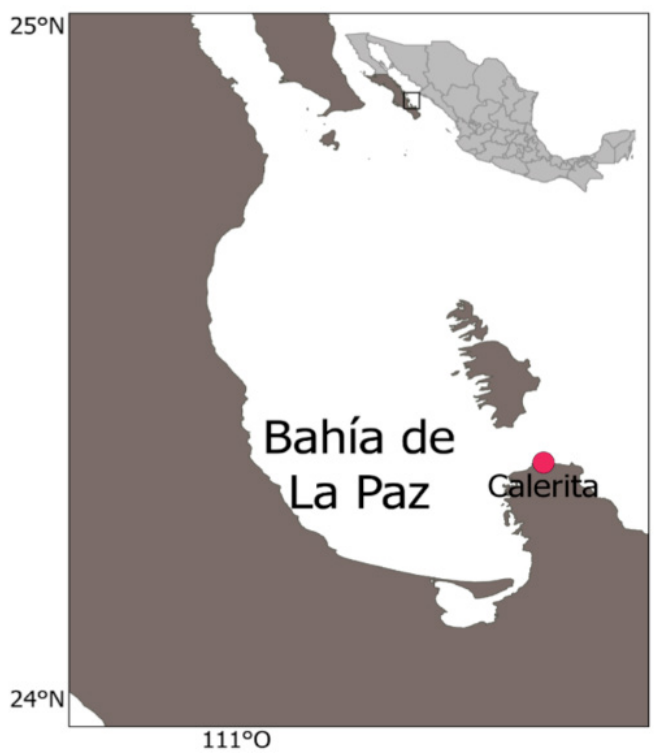

Andrea se esforzó en intentar descifrar de forma minuciosa las peculiaridades químicas de estos organismos, para poder relatar cómo su aparente delicadeza nace de una sólida microestructura. La curiosidad de Andrea la llevaría a buscar ayuda experta para ensamblar un rompecabezas de dimensiones micrométricas.

\section{Los carbonatos precipitados por la actividad fotosintética de las algas son biominerales formadores de costas}

Además de las técnicas químicas y el uso del microscopio electrónico, el uso de un dron para observar las pozas de marea también nos muestra cosas interesantes (ver figura 4). Por ejemplo, observamos que los manchones rojorosados, que son pequeños bosques de algas, cubren un área abundante de la costa. La nutrición de estas algas y los organismos de las pozas está basada en el agua marina de la Bahía de La Paz. Con estos nutrientes las algas realizan fotosíntesis, que es el proceso metabólico que les permite generar biomasa, tal como lo hacen las plantas.

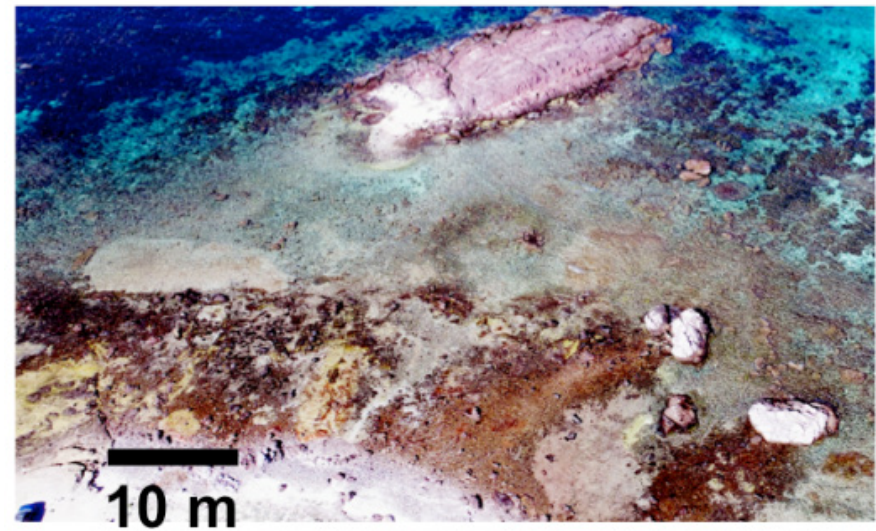


"Micromundos biominerales en las algas rojas" Patricia M. Valdespino, Andrea Bautista, Teresa Pi-Puig, Fabio Favoretto, Silvia Espinosa Matías, Hoi-Ying Holman y Anidia Blanco-Jarvio Vol. 22, Núm. 6, noviembre-diciembre 2021 Revista Digital Universitaria

Pero la fotosíntesis no sólo les sirve para crecer, también es un mecanismo que impulsa la formación de carbonatos en procesos fisicoquímicos en microescala, (Cabioch, 1988). Estos carbonatos se van endureciendo con el tiempo y constituyen los bloques estructurales de las costas. Los carbonatos tienen una morfología interna fascinante, que ha sido objeto de importantes investigaciones. Algunos conceptos fundamentales se pueden visitar aquí.

La difracción de rayos X (DRX) del material en polvo es sin duda la técnica más utilizada en la identificación mineralógica, debido a que los minerales presentan, por definición, una disposición ordenada de sus átomos, que se denomina estructura cristalina. Esta técnica se basa en la interferencia de los rayos X con la red cristalina de los minerales. Para el caso particular de los carbonatos biogénicos estudiados, la difracción de rayos X nos permitió identificar los polimorfos del carbonato de calcio (calcita o aragonita) y cuantificar la substitución del calcio por el magnesio (y otros elementos) en la estructura de la calcita. Además, cuantificamos con buena precisión todas las fases minerales cristalinas presentes (ver figura 5).

Figura 5. Ramita individual del alga y su composición mineral (Laboratorio de Difracción de RaYOS-X, LANGEM, UNAM).
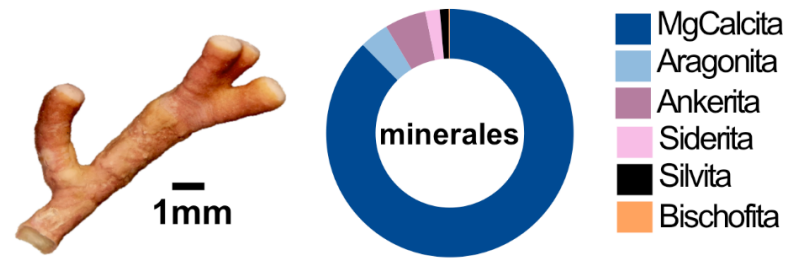

El esqueleto de las algas coralinas contiene aragonita, que es el biomineral que forman los corales. Además de aragonita, las algas coralinas forman carbonatos de calcio que atrapan magnesio en su estructura, llamados calcitas magnesianas. Si bien los resultados sobre la composición mineral de las algas estudiadas eran, en general, similares a los de algas coralinas de otras regiones, detectamos dos características particulares que llamaron nuestra atención.

La primera es que la calcita magnesiana del alga estudiada posee un alto contenido de Mg ( 18\%), y la segunda es la presencia de ankerita y siderita (carbonatos que contienen hierro) (Valdespino y et al., 2020). Este rasgo es muy interesante porque ahora sabemos que las calcitas con un contenido de magnesio relativamente alto (por ejemplo, mayor de 14\%) ofrecen mayor resistencia a la disolución, comparadas con calcitas con menos magnesio (Nash, 2013). Adicionalmente, la ankerita es un mineral que forma parte de las dolomitas, una familia de carbonatos reconocida por presentar una estructura con gran estabilidad química y térmica. En otras palabras, estas características detectadas podrían otorgar ventajas de resistencia a la disolución de estos carbonatos en un panorama de acidificación del océano.

\section{Luz más brillante para ver lo más pequeño}

Estudiar los micromundos de las algas rojas nos enseñó que la química y microestructura de sus biomateriales son en buena parte la clave de su resistencia estructural. Pero como suele suceder, estos interesantes resultados 
"Micromundos biominerales en las algas rojas" Patricia M. Valdespino, Andrea Bautista, Teresa Pi-Puig, Fabio Favoretto, Silvia Espinosa Matías, Hoi-Ying Holman y Anidia Blanco-Jarvio Vol. 22, Núm. 6, noviembre-diciembre 2021 Revista Digital Universitaria

inquietaron nuestra imaginación y nos llevaron a estudiar estos sistemas con un instrumento gigante que se usa para explorar lo más pequeño, un sincrotrón. En breve, es un acelerador de electrones que genera haces de luz muy brillante.

Nuestras primeras observaciones con la técnica sincrotrónica de espectromicroscopía de infrarrojo (Lawrence Berkeley National Laboratory) nos revelaron resultados muy interesantes. Pudimos averiguar que el esqueleto mineral no es homogéneo, ya que detectamos señales de distintos minerales en la cavidad de una célula del peritalo (o capa de crecimiento) del alga, que mide aproximadamente 20 micras de diámetro. También encontramos silicatos en el epitalo, que son minerales muy abundantes en nuestro planeta y que están formados por la unión de muchas unidades tetraédricas conformadas siempre por un átomo de silicio en el centro, enlazado con cuatro átomos de oxígenos en los vértices. El epitalo (ver figura 2) es una capa de unas cuantas micras que cubre la superficie del alga. De manera interesante, las algas se deshacen continuamente de esta capa, similarmente a la muda de piel de los reptiles. Esto les permite conservar una superficie adecuada para la fotosíntesis y otros procesos, ya que como puedes observar en la fotografía del epitalo, allí se acumulan partículas y otros organismos.

En resumen, hay un interés creciente en las algas como candidatos vivos para secuestrar el carbono atmosférico en estructuras estables como los biominerales. El estudio de la formación de carbonatos en las algas de la Bahía de La Paz reveló algunas claves de la resistencia estructural de estos organismos y de su papel como fuente de materiales (carbonatos y silicatos), que con el tiempo formarán parte de las costas. Los retos de esta investigación son un motor de ciencia básica y pueden contribuir a inspirar nuevas estrategias para mitigar el cambio climático.

\section{Referencias}

- Bautista, A. (2020). Diversidad estacional de procariontes y caracterización química de algas coralinas intermareales de la Bahía de la Paz [Tesis de Maestría. Posgrado en Ciencias Marinas y Costeras. Universidad Autónoma de Baja California Sur].

* Cabioch, J. (1988). Morphogenesis and generic concepts in coralline algae-a reappraisal. Helgoländer Meeresuntersuchungen, 42(3), 493-509. https://doi. org/10.1007/BF02365623

- Nash, M. C., Opdyke, B. N, Troitzsch, U., Russell, B. D., Adey, W. H., Kato, A., DiazPulido, G., Brent, C., Gardner, M., Prichard, J. y Kline D. I. (2013). Dolomite-rich coralline algae in reefs resist dissolution in acidified conditions. Nature climate change 3(3), 268-272. https://doi.org/10.1038/nclimate1760 
"Micromundos biominerales en las algas rojas" Patricia M. Valdespino, Andrea Bautista, Teresa Pi-Puig, Fabio Favoretto, Silvia Espinosa Matías, Hoi-Ying Holman y Anidia Blanco-Jarvio Vol. 22, Núm. 6, noviembre-diciembre 2021 Revista Digital Universitaria

- Valdespino-Castillo, P. M., Bautista-Garcia, A., Favoretto, F., Merino-Ibarra, M., Alcántara-Hernández, R. J., Pi-Puig, T., Espinosa-Matías, S., Castillo, F. S., Holman, $\mathrm{H}-\mathrm{Y}$. y Blanco-Jarvio, A. (2020). Interplay of microbial communities with mineral environments in coralline algae. Science of the Total Environment, 757,143877. https://doi.org/10.1016/j.scitotenv.2020.143877

* Ziveri. Acidificación del océano [presentación en diapositivas]. uson. https://sivea. uson.mx/docentes/tareas/16_PRECIPITAClyiN_CARBONATOS.pdf

\section{Cómo CITAR ESTE ARTículo}

- Valdespino, Patricia M., Bautista, Andrea, Pi-Puig, Teresa, Favoretto, Fabio, Espinosa Matías, Silvia, Holman, Hoi-Ying y Blanco-Jarvio, Anidia. (2021, noviembrediciembre). Micromundos biominerales en las algas rojas. Revista Digital Universitaria (RDU), 22(6). http://doi.org/10.22201/cuaieed.16076079e.2021.22.6.4 